\title{
Mid-IR direct imaging of superjupiters around nearby stars
}

\author{
Gabriel Bihain ${ }^{1,2}$, Rafael Rebolo ${ }^{1,2}$, \\ José Antonio Caballero ${ }^{1}$ \\ and Victor Javier Sánchez Béjar ${ }^{1,3}$ \\ ${ }^{1}$ Instituto de Astrofísica de Canarias, 38205 La Laguna, Tenerife, Spain \\ ${ }^{2}$ Consejo Superior de Investigaciones Científicas, Spain \\ ${ }^{3}$ Gran Telescopio Canarias Project, Spain \\ email: gbihain@ll.iac.es
}

\begin{abstract}
Superjupiters are much brigther when they are young and still contracting, and the contrast with the harboring stars is significantly more favorable for their detection. Within the JOVIAN (Jupiter-like Objects in the Visible and the Infrared: Astrophysical Nature) project, a search has been performed for superjupiters around nearby young stars using $4 \mathrm{~m}$ class telescopes. Here we summarize the results and present prospects for a future search around young and also relatively older nearby stars with the $10 \mathrm{~m}$ Gran Telescopio Canarias and the mid-infrared camera CanariCam.
\end{abstract}

Keywords. (Galaxy:) solar neighborhood, stars: low-mass, (stars:) planetary systems.

\section{Introduction}

The sensitivity to detect superjupiters around stars improves when the spatial resolution is higher (i.e. for nearby stars) and the contrast is lower (i.e. for primaries that are low-mass stars and planets that are intrinsically brighter due to youth). Numerous searches for massive planets by direct near-infrared (IR) imaging around nearby young dwarfs have been performed in the past 10 years (Nakajima, Oppenheimer, Kulkarni, et al. 1995; Oppenheimer, Golimowski, Kulkarni, et al. 2001; McCarthy \& Zuckerman 2004; Luhman \& Jayawardhana 2002; Neuhäuser, Guenther, Alves, et al. 2003; Masciadri, Mundt, Henning, et al. 2005; Lowrance, Patrick, Becklin, et al. 2005). Only two good planet candidates around dwarfs have been discovered so far (Chauvin, Lagrange, Dumas, et al. 2004,2005; Neuhäuser, Guenther, Wuchterl, et al. 2005). The very paucity observed of exoplanets at large- to moderate separations ( $>10 \mathrm{AU})$ can provide valuable indications on the main formation process of these objects, supposing that their intrinsic evolution (and thus photometric and spectroscopic properties) are well enough predicted by theoretical models (e.g. Baraffe, Chabrier, Allard, et al. 2002; Burrows, Sudarsky \& Lunine 2003). We summarize the effort made by our group to search for substellar companions using Adaptive Optics (AO) systems and present our plan to exploit the advantages offered in the mid-IR by high planet/star contrast, diffraction-limited and subarcsecond imaging with a $10 \mathrm{~m}$ class telescope.

\section{Adaptive optics searches}

Using Alfa+Omega-Cass at the 3.5-m Calar Alto, AdOpt+NICS at Telescopio Nazionale Galileo and, especially, NAOMI+INGRID at the $4.2 \mathrm{~m}$ William Herschel Telescope (WHT), Caballero et al. (in preparation) have searched for substellar companions of more than 


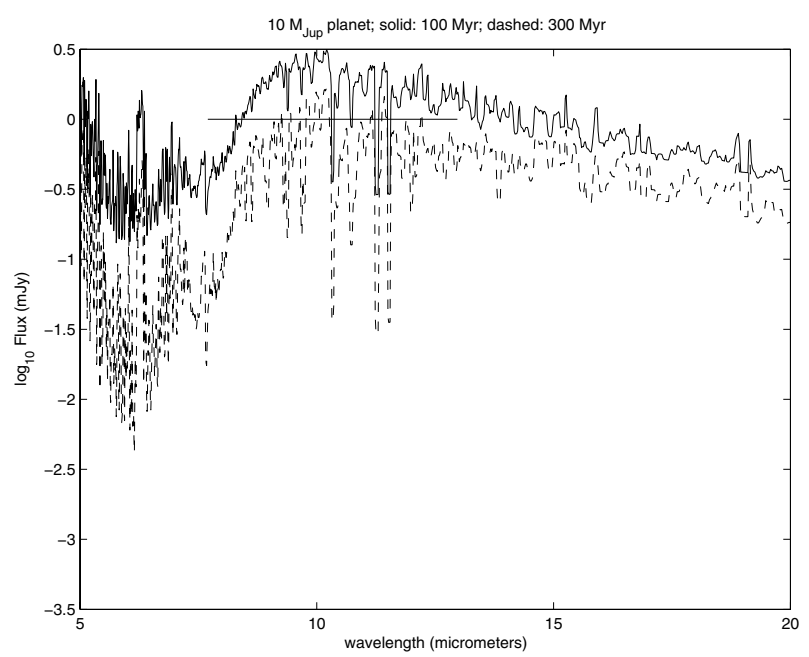

Figure 1. The expected mid-IR spectral energy distributions for a $10 \mathrm{M}_{\mathrm{Jup}}$ planet at $10 \mathrm{pc}$, for ages of 100 and $300 \mathrm{Myr}$ (solid and dashed lines, respectively; Burrows et al. 2003). The horizontal line corresponds to the CanariCam $25 \sigma \mathrm{N}$-band sensitivity in $30 \mathrm{~min}$ of on-source integration.

fifty young nearby stars $(\mathrm{d}<50 \mathrm{pc})$ in the near-IR. The stars were selected with spectral types later than solar and features indicative of youth (high lithium abundance, X-ray and/or UV emission, membership to young proper motion associations, etc.). Their ages ranged between 15 and $600 \mathrm{Myr}$. All of them have been completely analysed, comparing first and second astrometric epochs. Although the data would allow them to discover objects with masses down to $5 \mathrm{M}_{\text {Jup }}$ at separations of 4 arcsec around several of the primaries (i.e. at $40 \mathrm{AU}$ for the nearest stars), they have not detected any new substellar companion up to $\sim 1000 \mathrm{AU}$. This result implies a frequency of substellar companions at intermediate and large separations of $<4 \%$, in agreement with the $1 \pm 1 \%$ obtained by McCarthy \& Zuckerman (2004). It should also be compared with the $7.3 \pm 1.5 \%$ of the solar-like stars that have exoplanet candidates discovered at small separations with the radial velocity method.

\section{Mid-infrared searches}

We plan to perform imaging at $10 \mu \mathrm{m}$ with the $10 \mathrm{~m}$ Gran Telescopio Canarias (GTC)/ CanariCam and with enough sensitivity ( $0.2 \mathrm{mJy}$ at $5 \sigma$ in $30 \mathrm{~min}$ on-source integration) to detect planetary companions of masses $10 \mathrm{M}_{\mathrm{Jup}}$ around stars of the solar neighborhoud. In Fig. 1 and 2 we illustrate the sensitivity of this instrument. The subarsecond imaging provided by CanariCam will permit us to explore physical separations as small as $10 \mathrm{AU}$ around targets at $10 \mathrm{pc}$ (approximately five times the telescope resolving power $0.2 \operatorname{arcsec})$.

In addition to the diffraction limited imaging in the mid-IR, we can take advantage of the improved planet/star brightness contrast. For example, a $10 \mathrm{M}_{\mathrm{Jup}}$ superjupiter at $10 \mathrm{pc}$ and $100 \mathrm{Myr}$ old has a predicted mid-IR magnitude $N \sim 10.5$ and a near-IR magnitude $J \sim 20$, whereas its K0-type harboring star would have $N \sim J \sim 4$. Therefore the contrast planet/star in magnitude decreases from 16 to 6.5 magnitudes when shifting to the mid-IR, and thus enters in the typical instrumental dynamical range for detection. 


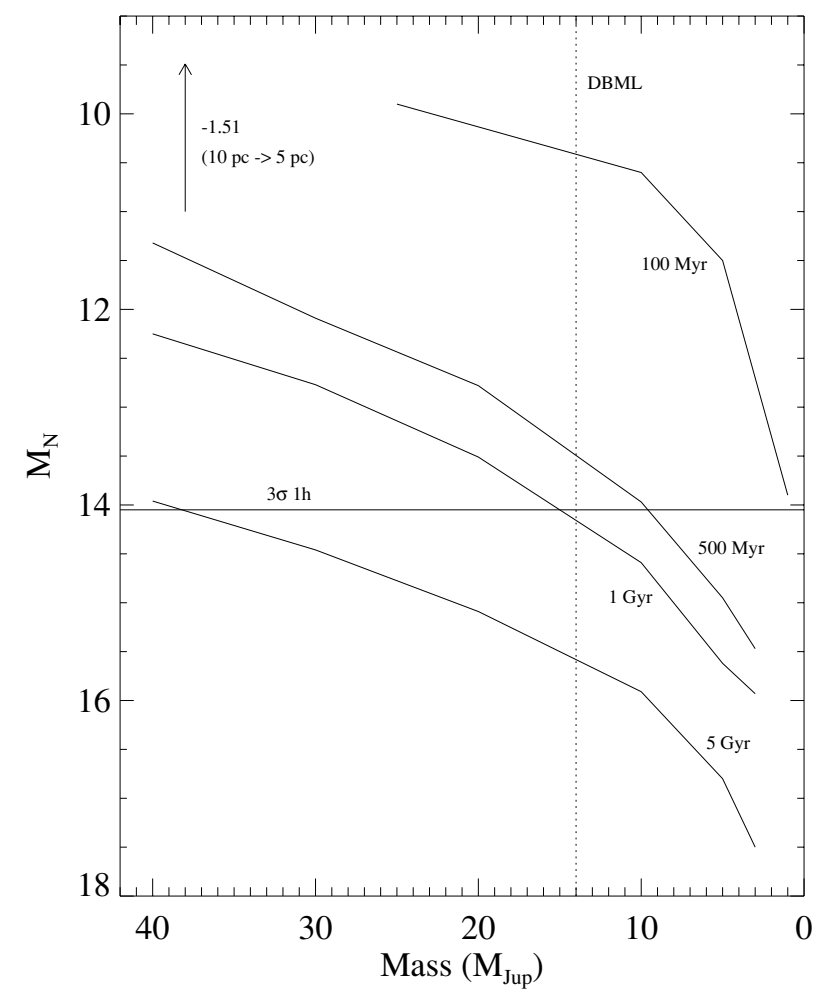

Figure 2. 5- $\sigma N$-band sensitivity of CanariCam in 1 hour of on-source integration. Theoretical isochrones from Burrows, Sudarsky, Lunine, et al. (1998) at ages 0.1, 0.5, 1 and 5 Gyr for $\sim 1-40 \mathrm{M}_{\text {Jup }}$ substellar objects at $10 \mathrm{pc}$ are overploted (solid lines). The arrow indicates the brightness increment when these objects are at $5 \mathrm{pc}$. Finally the deuterium mass burning limit (DMBL) is traced by the vertical dotted line.

With GTC/Canaricam we will be able to search three types of $10 \mathrm{M}_{\mathrm{Jup}}$ superjupiters (see Fig. 2) of different ages and distances: (i) $100 \mathrm{Myr}$ at $<50 \mathrm{pc}$; (ii) $1 \mathrm{Gyr}$ at $<10 \mathrm{pc}$; (iii) 5 Gyr at $<5$ pc. Objects at $5 \mathrm{pc}$ are $1.5 \mathrm{mag}$ brighter than at $10 \mathrm{pc}$, and objects at $50 \mathrm{pc}$ are $3.5 \mathrm{mag}$ fainter than at $10 \mathrm{pc}$. For case $(\mathrm{iii})$, there are approximately 60 stellar targets available for the GTC in the North Hemisphere and some would require integration times up to 2.5 hours. Direct mid-IR imaging of stellar systems spanning an age range of 0.1-5 Gyr will permit to gain further insight on the formation and evolution processes of planetary mass objects.

\section{Conclusions}

The near-IR Adaptive Optics searches that we have performed with 4 m-class-telescopes provided no detection of massive planets at large separations from 15-600 Myr-old nearby stars. In the mid-IR, higher planet/star contrast can be obtained than in the near-IR, and also diffraction-limited images. We plan to search for superjupiters in the mid-IR with the GTC/CanariCam. For 5 Gyr old stars at less than 5 pc, superjupiters of $10 \mathrm{M}_{\text {Jup }}$ could be discovered at separations of $\sim 5 \mathrm{AU}$.

\section{References}

Baraffe, I., Chabrier, G., Allard, F., \& Hauschildt, P.H. 2002, A $\mathscr{\mho} A$ 382, 563 
Burrows, A., Sudarsky, D., Sharp, C., Marley, M.S., Hubbard, W.B., Lunine, J.I., Guillot, T., Saumon, D., \& Freedman, R. 1998, ASPC 134, 354

Burrows, A., Sudarsky, D. \& Lunine, J.L. 2003, ApJ 596, 587

Chauvin, G., Lagrange, A.-M., Dumas, C., Zuckerman, B., Mouillet, D., Song, I., Beuzit, J.-L., \& Lowrance, P. 2004, A\& A 425, L29

Chauvin, G., Lagrange, A.-M., Dumas, C., Zuckerman, B., Mouillet, D., Song, I., Beuzit, J.-L., \& Lowrance, P. 2005, A\&A 438, L25

Lowrance, P.J., Becklin, E.E., Schneider, G., Kirkpatrick, J.D., Weinberger, A.J., Zuckerman, B., Dumas, C., Beuzit, J.-L., Plait, P., Malumuth, E., Heap, S., Terrile, R.J., \& Hines, D.C. 2005, AJ 130, L1845

Luhman, K.L., Jayawardhana, R. 2002, ApJ 566, L1132

Masciadri, E., Mundt, R., Henning, Th., Alvarez, C., \& Barrado y Navascués, D. 2005, ApJ 625,1004

McCarthy, C. \& Zuckerman, B. 2004, AJ 127, 2871

Neuhäuser, R., Guenther, E.W., Alves, J., Huélamo, N., Ott, Th., \& Eckart, A. 2003, AN 324, 535

Neuhäuser, R., Guenther, E.W., Wuchterl, G., Mugrauer, M., Bedalov, A., \& Hauschildt, P.H. 2005, A\&A 435, L13

Nakajima, T., Oppenheimer, B.R., Kulkarni, S.R., Golimowski, D.A., Matthews, K., \& Durrance, S.T. 1995, Nature 378, 463

Oppenheimer, B.R., Golimowski, D.A., Kulkarni, S.R., Matthews, K., Nakajima, T., CreechEakman, M., Durrance, S.T. 2001, AJ 121, 2189 